\title{
INESTABILIDAD GLENOHUMERAL: LO QUE EL RADIOLOGO DEBE SABER
}

Dras. Carolina Zamorano C(1), Sara Muñoz Ch$^{(2)}$, Paola Paolinelli $G^{(2)}$.

1. Radióloga. Alumna Post grado Universidad de Chile.

2. Radióloga. Diagnóstico por Imágenes Clínica Las Condes. Profesor Agregado Universidad de Chile.

\section{GLENOHUMERAL INSTABILITY: WHAT THE RADIOLOGIST SHOULD KNOW}

\begin{abstract}
Glenohumeral instability is a common cause of pain and functional limitation of the shoulder, which involves symptomatic subluxation or dislocation of the humeral head with respect to the glenoid fossa. Glenohumeral instability may be classified according to several parameters: degree, direction, timing, etiology and biomechanics of the dislocation, among others. Imaging methods play an important role in the evaluation of glenohumeral instability, being all of them useful, complementary, and not necessarily mutually exclusive modalities. The following article presents a review of the main types of glenohumeral instability and related imaging findings.
\end{abstract}

Keywords: Glenoid fossa, Glenohumeral instability, Humeral head, Symptomatic dislocation.

Resumen: La inestabilidad glenohumeral es una causa frecuente de dolor y limitación funcional del hombro, que implica subluxación o luxación sintomática de la cabeza humeral con respecto de la fosa glenoidea. Puede clasificarse considerando varios aspectos: grado, dirección, cronología, etiología y biomecánica de la luxación, entre otros. La imaginología juega un rol importante en la evaluación de la inestabilidad glenohumeral, siendo todos los métodos de imágenes útiles, complementarios entre sí y no necesariamente excluyentes. En el siguiente artículo presentamos una revisión de los principales tipos de inestabilidad glenohumeral y los hallazgos imaginológicos asociados.

Palabras clave: Cabeza humeral, Fosa glenoidea, Inestabilidad glenohumeral, Luxación sintomática.

Zamorano $\boldsymbol{C}$ y cols. Inestabilidad glenohumeral: Lo que el radiólogo debe saber. Rev Chil Radiol 2009; 15:

Correspondencia: Carolina Zamorano $C$. carolinazamorano@vtr.net

Trabajo recibido el 02 de julio de 2009, aceptado para publicación el 11 de agosto 2009.

\section{Introducción}

La luxación glenohumeral es la más frecuente de las luxaciones, aproximadamente $50 \%$ del total de las luxaciones. La discrepancia de tamaño entre la cavidad glenoidea y la cabeza humeral otorga a la articulación un gran rango de movilidad, pero le confiere además una especial vulnerabilidad a la luxación ${ }^{(1,2)}$.

El 95\% de las luxaciones glenohumerales son de tipo anterior y de ellas la mayor parte (entre el 50$70 \%$ ) se presenta en pacientes menores de 30 años y afecta con mayor frecuencia a hombres. Clínicamente, el paciente con el hombro luxado resiste el mínimo intento de movilidad pasiva y la abducción activa del brazo, observándose asimetría en el hombro. El acromion se hace prominente y la cabeza humeral puede palparse anteriormente.

$\mathrm{La}$ inestabilidad glenohumeral es una causa frecuente de dolor y de limitación funcional en el hombro. Implica pérdida de la relación articular entre la cabeza humeral y la cavidad glenoidea. Puede ser clasificada de acuerdo a varios criterios; recientemente se ha propuesto una clasificación clínico-radiológica basada en la etiología y que considera los hallazgos imaginólogicos ${ }^{(1-3)}$.

La imaginología juega un rol importante en la evaluación de la inestabilidad glenohumeral, siendo útiles todos sus métodos de estudio, complementarios entre sí y no necesariamente excluyentes. Como sucede en la evaluación de muchas otras patologías, el estudio imaginológico de la inestabilidad debe comenzar siempre con la radiografía simple, especialmente útil en la detección de la mayoría de las lesiones óseas producidas como consecuencia de la luxación y de las alteraciones morfológicas articulares que pueden predisponer a ella.

El ultrasonido (US) es una herramienta útil, ya que es un método ampliamente disponible y de bajo costo; sin embargo, en la evaluación de la inestabilidad glenohumeral su papel se limita al estudio de los tendones del manguito rotador y no contribuye al diagnóstico de las lesiones propias de la inestabilidad, especialmente las que comprometen al complejo 
cápsulo-labral.

La tomografía computada (TC) es útil en la evaluación de la morfología y lesiones de las estructuras óseas. Cuando se utiliza en combinación con la inyección de contraste intraarticular (artro-TC), sirve además para la evaluación de los tejidos blandos intraarticulares, tales como complejo cápsulo-labral, labro-bicipital y ligamentos. Se ha comenzado a utilizar además para la valoración de la magnitud de las lesiones óseas, tanto de la glenoides como de la cabeza humeral, ya que permite hacer una estimación más exacta de la superficie ósea perdida tras uno o varios episodios de luxación.

La resonancia magnética (RM) y especialmente la artro-RM, son las técnicas de elección en la caracterización de lesiones propias de la inestabilidad glenohumeral, tales como desgarros del complejo labrobicipital, cápsulo-labral y del intervalo de los rotadores, así como también en la evaluación de entidades relacionadas, como el pinzamiento pósterosuperior. Cabe señalar la utilidad de la RM cuando se realiza en la fase aguda de la luxación, habitualmente después de la reducción ortopédica, pues en la mayoría de estos casos hay derrame articular que determina un efecto artrográfico espontáneo al producir una distensión capsular, necesaria para la evaluación de las estructuras intraarticulares que frecuentemente resultan dañadas.

\section{Estabilidad glenohumeral}

La estabilidad funcional de la articulación glenohumeral puede ser definida como la mantención del alineamiento del centro de la cabeza humeral dentro de la cavidad glenoidea durante el movimiento, logrado mediante la sincronización precisa de mecanismos estáticos (pasivos) y dinámicos (activos). El término laxitud se refiere a la traslación pasiva y asintomática de la cabeza humeral ${ }^{(1-5)}$, a diferencia del concepto de inestabilidad que implica subluxación o luxación sintomática de la cabeza humeral respecto a la cavidad glenoidea.

Los mecanismos estáticos o pasivos incluyen: presión intraarticular negativa, adhesión y cohesión de las superficies articulares, tamaño, forma y orientación de la fosa glenoidea y el complejo cápsulo-labral(6).

Los mecanismos dinámicos o activos incluyen: los tendones del manguito rotador y el tendón de la porción larga del bíceps ${ }^{(6)}$.

El mecanismo cápsulo-labral es el mecanismo más importante en la estabilización de la articulación glenohumeral; a su vez se divide en mecanismo capsular anterior y posterior. Los componentes que participan en el mecanismo capsular anterior son: aspecto anterior de la cápsula fibrosa, ligamentos glenohumerales, membrana sinovial y sus recesos, el labrum glenoideo anterior, músculo y tendón subescapular y periostio anterior de la escápula. En el mecanismo capsular posterior se incluye el aspecto posterior de la cápsula fibrosa, membrana sinovial posterior, labrum glenoideo posterior, periostio posterior de la escápula y el manguito rotador pósterosuperior (tendones y músculos supraespinoso, infraespinoso y redondo menor) ${ }^{(6)}$ (Figura 1).

El tendón de la cabeza larga del bíceps, el ligamento coracohumeral, el ligamento coracoacromial y el tendón del tríceps constituyen un soporte adicional a las estructuras de la articulación glenohumeral. EI manguito rotador y la coordinación escápulo-torácica son mecanismos significativos en mantener la estabilidad articular durante el movimiento ${ }^{(6)}$ (Figura 2).

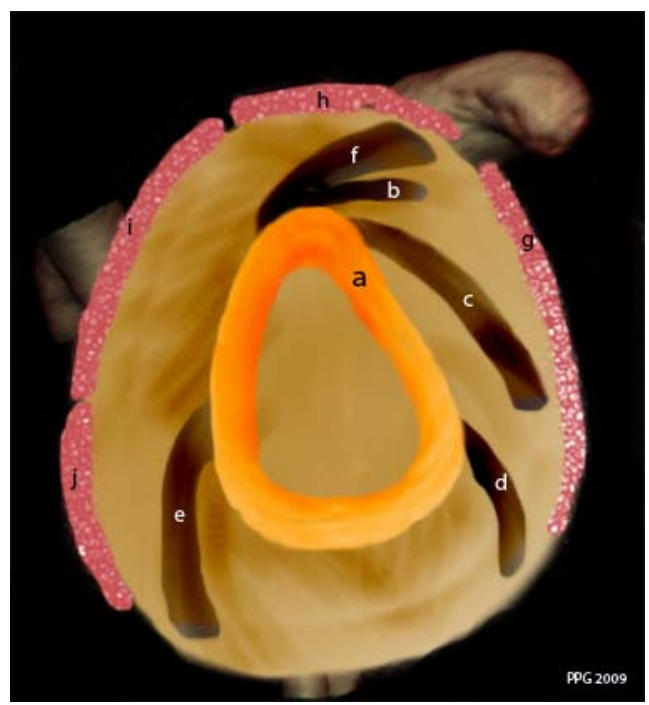

Figura 1a. Esquema de corte sagital: a labrum. b: ligamento glenohumeral (LGH) superior. c: LGH medio. d-e: LGH inferior. f: Tendón largo del bíceps. g: tendón (T.) subescapular. $h$ : T. supraespinoso. $i$ : T. infraespinoso j: T. redondo menor.

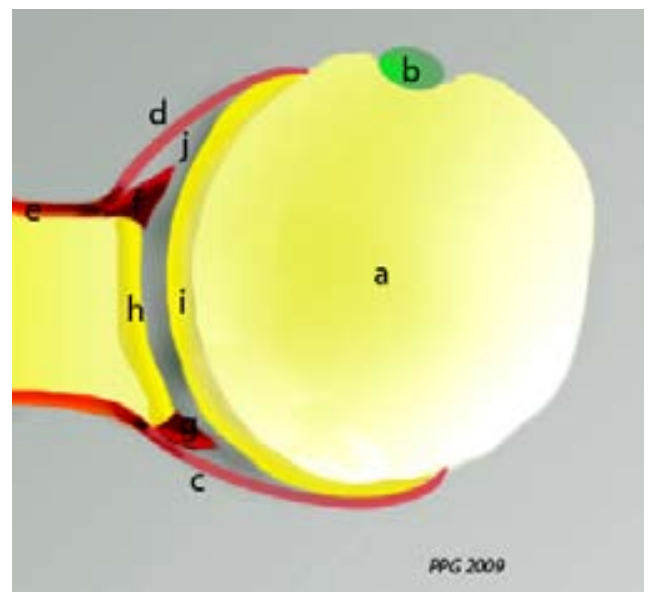

Figura 1b. Esquema de corte axial. a: cabeza humeral. b: T. bíceps c: cápsula posterior. d: complejo capsular anterior e: periostio. f: labrum anterior. g: labrum posterior. h: cartílago hialino glenoideo. i: cartílago humeral. j: espacio articular. 
AVISO 
AVISO 


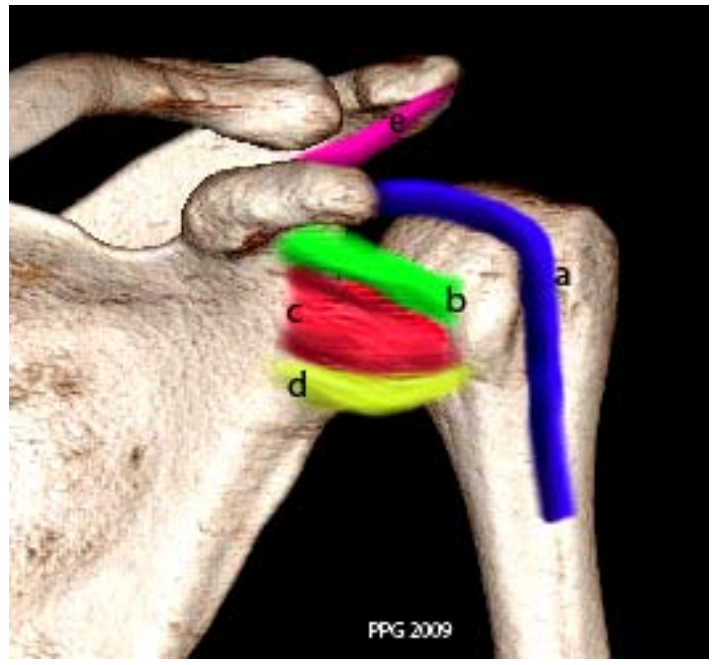

Figura 2a. a: T bíceps. b: LGH superior. c: $L G H$ medio. d: LGH inferior. e: Ligamento coracoacromial.

La inestabilidad glenohumeral implica pérdida de la relación articular entre la cabeza humeral y la cavidad glenoidea. Es una causa frecuente de dolor y limitación funcional y puede ser clasificada de acuerdo a: grado (luxación o subluxación), dirección (anterior, posterior o multidireccional), cronología (aguda o crónica), etiología (traumática, atraumática o microtraumática) y biomecánica (estática, dinámica o voluntaria) ${ }^{(1-3,6)}$. Recientemente se ha propuesto una clasificación clínico-radiológica de la inestabilidad, basada fundamentalmente en la etiología, la dirección de ésta y algunos hallazgos imaginólogicos ${ }^{(6)}$ (Tabla I y II).

\begin{tabular}{|ll|}
\hline Tabla I. & \\
TUBS & AMBRI \\
T: traumatic & A: atraumatic \\
(episodio traumático) & (etiología atraumática) \\
U: Unidireccional & M: multidireccional \\
B: Bankart & B: bilateral \\
$\quad$ (lesión del labrum & (generalmente compro \\
ánteroinferior) & mete ambos hombros) \\
S: Surgery & R: rehabilitación \\
(requiere cirugía & (buena respuesta \\
correctiva) & a este tratamiento) \\
& I: intervalo \\
& (puede asociarse a lesión \\
& del intervalo)
\end{tabular}

Tabla II.
AIOS
A: adquirida
I: inestabilidad
O: overstress (sobreuso)
S: Surgery (requiere cirugía correctiva)

Puede incluirse la inestabilidad microtraumática desarrollada en los deportistas "lanzadores".
Tipos de inestabilidad glenohumeral y sus principales hallazgos imaginológicos

En la mayoría de los casos, la inestabilidad es de causa traumática y su dirección ánteroinferior, constituyendo aproximadamente el $95 \%$ del total de casos. Es menos frecuente la inestabilidad traumática posterior y la inestabilidad atraumática multidireccional, siendo en conjunto el $5 \%$ de todas las luxaciones ${ }^{(1,2,6)}$

Un nuevo tipo de inestabilidad, la denominada microinestabilidad, ha recibido atención en la literatura ortopédica en los últimos años ${ }^{(1,2,6)}$.

\section{Luxación ánteroinferior}

Generalmente es secundaria a traumatismo agudo y constituye la forma más frecuente de luxación traumática, aproximadamente el $95 \%$ de todos los $\operatorname{casos}^{(2,7)}$

Los pacientes pueden presentarse clínicamente con lesiones óseas (Bankart y HillSachs), lesiones ligamentosas, capsulares y/o condrales, así como quistes paralabrales, cuerpos intraarticulares y roturas del manguito rotador.

\section{Lesiones óseas}

\section{a. Lesión de Hill-Sachs}

Corresponde a una fractura por compresión con impactación del margen pósterolateral de la cabeza humeral. Son fáciles de identificar en imágenes radiográficas y en RM convencional (Figuras 3, 4). Un estudio de artro-RM debería incluir secuencias sensibles al líquido, con el objeto de identificar edema óseo, que traduzca una contusión ósea reciente (Figuras 5,6). Grandes deformidades de Hill-Sachs pueden tener un valor pronóstico, al aumentar la probabilidad de recurrencias.

\section{b. Bankart óseo}

Es una fractura del margen ánteroinferior de la glenoides. Puede aparecer consolidada o evolucionar como falta de unión ${ }^{(8)}$.

En inestabilidad crónica, esta lesión puede asociarse a irregularidad del margen glenoideo ánteroinferior, lo que determinaría potencialmente una restricción en la congruencia articular (Figura 7).

\section{c. Glenoides en "pera invertida"}

Este tipo morfológico de glenoides predispone a la luxación anterior. Se visualiza mejor en la proyección outlet del estudio radiológico y en las imágenes sagitales de TC y RM. Su identificación es importante, por cuanto puede tener implicancia quirúrgica ${ }^{(9)}$.

\section{d. Otras lesiones óseas}

En pacientes de mayor edad, la luxación traumática puede asociarse con fractura por avulsión de la tuberosidad mayor. 


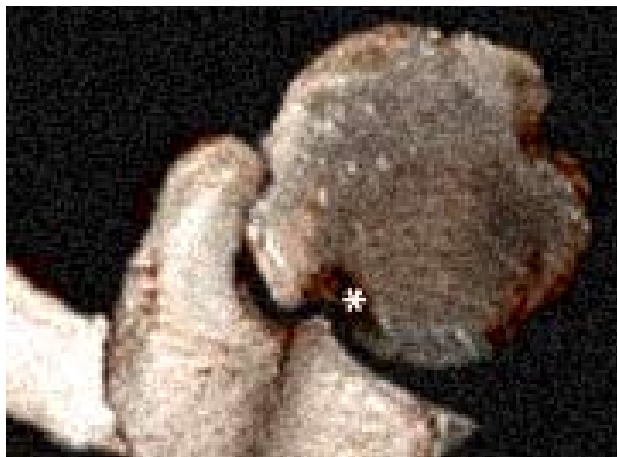

Figura 3. Lesión Hill Sachs: En la luxación anterior la cabeza se impacta en el reborde glenoideo anterior lo que produce una fractura por hundimiento en el aspecto pósterolateral de la cabeza humeral ( $\left.{ }^{*}\right)$.

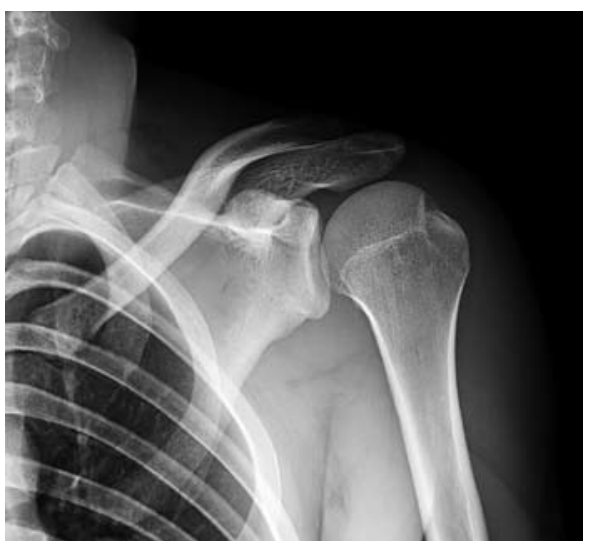

Figura 4 a. $R X$ AP de hombro. Fractura de Hill Sachs.

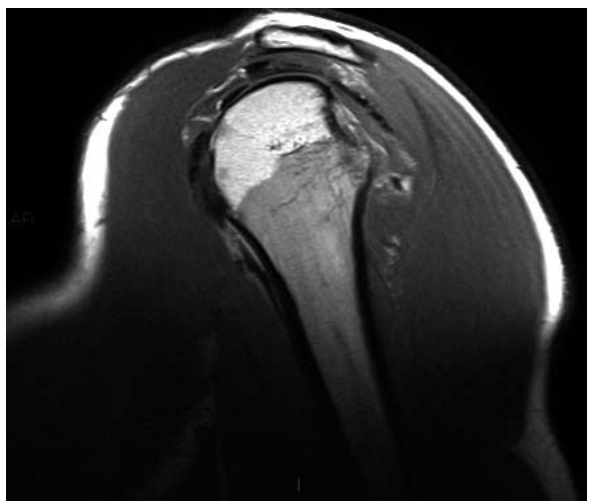

Figura 4 b. $R M$ corte sagital T1. Fractura de Hill Sachs.

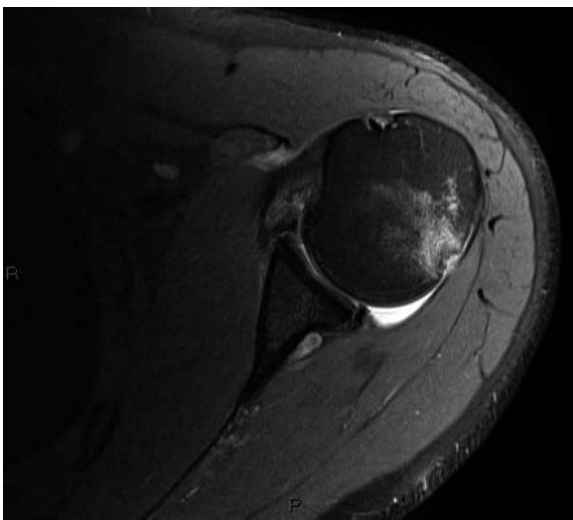

Figura 5 a. $R M$ corte axial $D P$ con saturación de la grasa: Edema óseo en el aspecto pósterolateral de la cabeza humeral.
Figura 5 b. $R M$ corte sagital DP con saturación de la grasa. El mismo paciente de la figura anterior.

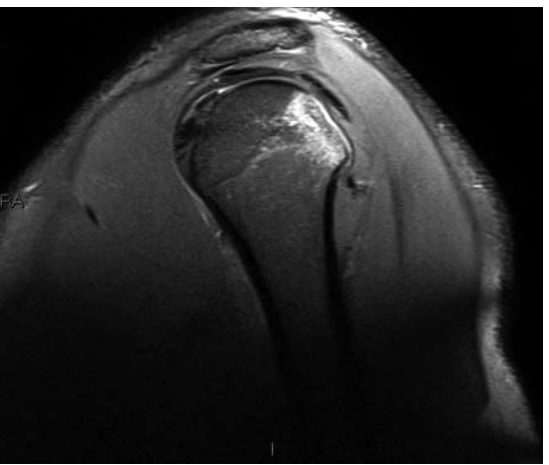

Figura 6 a. $R M$ corte axial DP con saturación de la grasa. Gran defecto por hundimiento en aspecto pósterolateral de la cabeza humeral. Fractu ra de Hill Sachs.

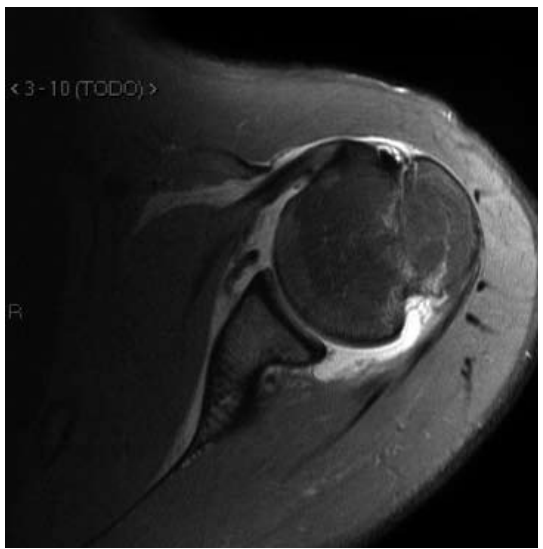

Figura 6 b. $R M$ corte sagital DP con saturación de la grasa. El mismo paciente de la figura anterior.
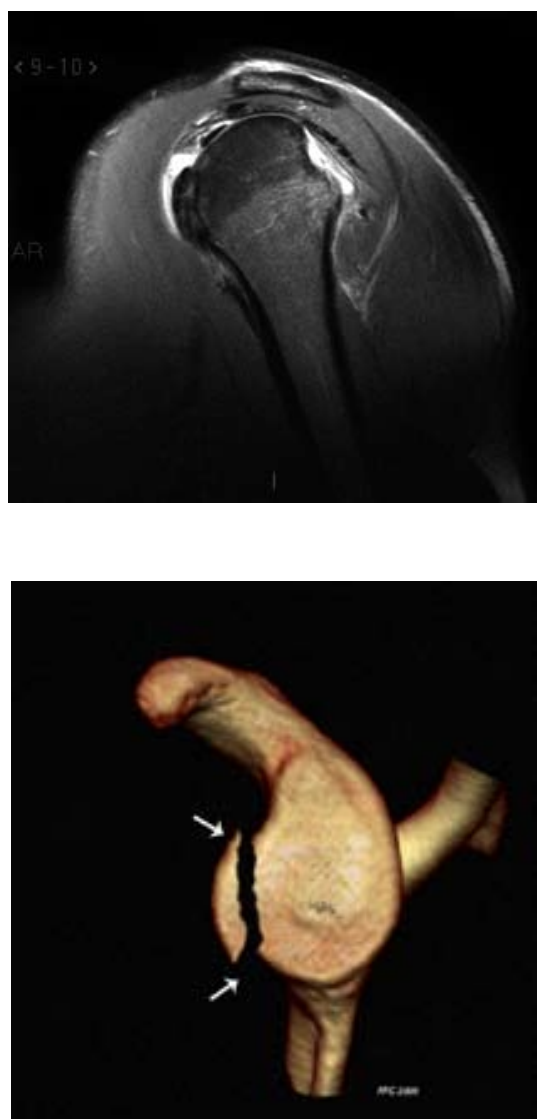

Figura 7. Lesión de Bankart óseo: Fractura de aspecto anterior del reborde glenoideo. 


\section{Lesiones de partes blandas tipo Bankart y sus variantes}

Incluyen un amplio espectro de lesiones traumáticas que pueden afectar al labrum, periostio, cápsula articular, ligamentos glenohumerales y/o cartílago articular. La RM y especialmente la artroRM son el método diagnóstico de elección para estas lesiones ${ }^{(8,19,20)}$.

\section{a. Lesión de Bankart clásica}

Consiste en la rotura o desinserción del labrum ánteroinferior, con desgarro asociado del periostio $(8,19,20)$. En la evaluación con RM es más evidente en posición de abducción y rotación externa (ABER). Puede también existir desgarro de todo el labrum anterior (Figuras 8, 9).

b. Variantes de la lesión de Bankart

Existen dos importantes variantes de lesión de Bankart, que incluyen lesión tipo ALPSA y lesión tipo Perthes.

- ALPSA (anterior labroligamentous periosteal sleeve avulsion)

Consiste en desgarro del labrum y del ligamento glenohumeral inferior, asociados a desprendimiento del periostio con integridad del mismo, que se desplazan y rotan medial e inferiormente, originando un tejido cicatricial que finalmente puede sinovializarse (Figura 10)(8, 19, 20).

- Perthes

El labrum desgarrado puede aparecer normalmente situado, aunque funcionalmente comprometido. El periostio escapular anterior se observa despegado, pero íntegro (Figura 11) ${ }^{(8,19,20)}$.

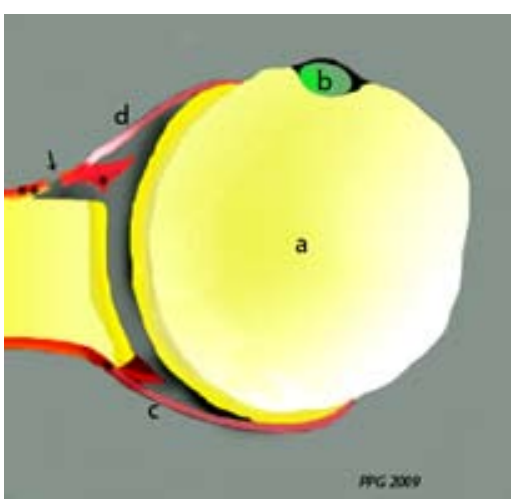

Figura 8 a. Esquema de Bankart blando: a: cabeza humeral. $b$ : T. bíceps. c: cápsula posterior. d: complejo capsular anterior. Desprendimiento del labrum anterior $\left.{ }^{*}\right)$ desde el reborde glenoideo, con rotura del periostio $\left({ }^{\star *}\right)$.

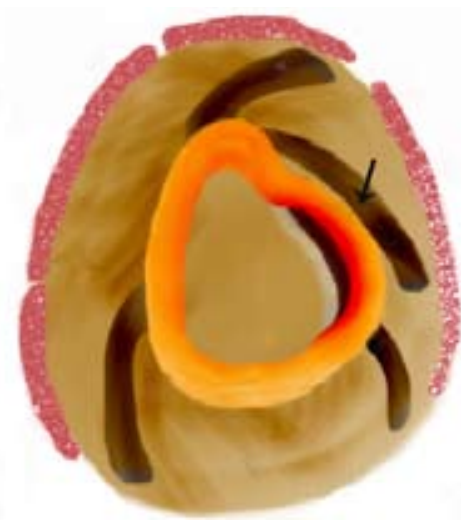

Figura 8 b. Localización de lesiones de Bankart $y$ variantes.
Figura 9 a. $R M$ corte axial GE T2. Lesión de Bankart.

Figura 9 b. $R M$ corte sagital $D P$ con saturación de la grasa. Lesión de Bankart.

Figura 10 a. Esquema lesión ALPSA: Desprendimiento del labrum anterior desde el gle-noides, con desplazamiento medial (*).
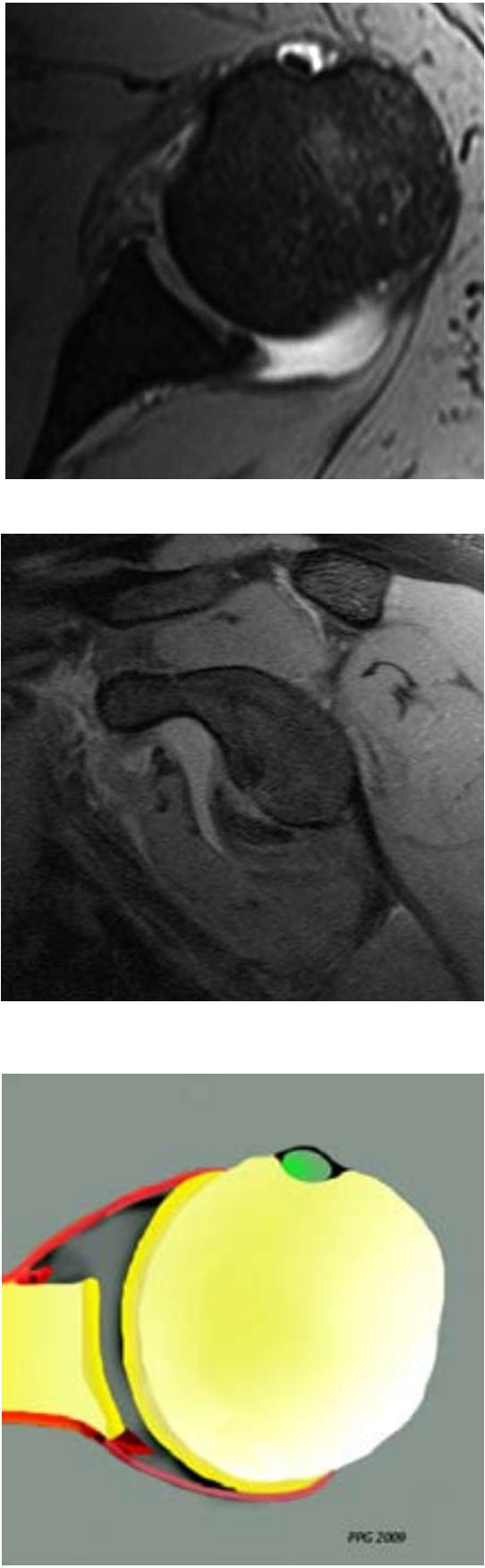

Figura 10 b. $R M$ corte axial DP con saturación de la grasa. Hemartrosis. Lesión ALPSA. 

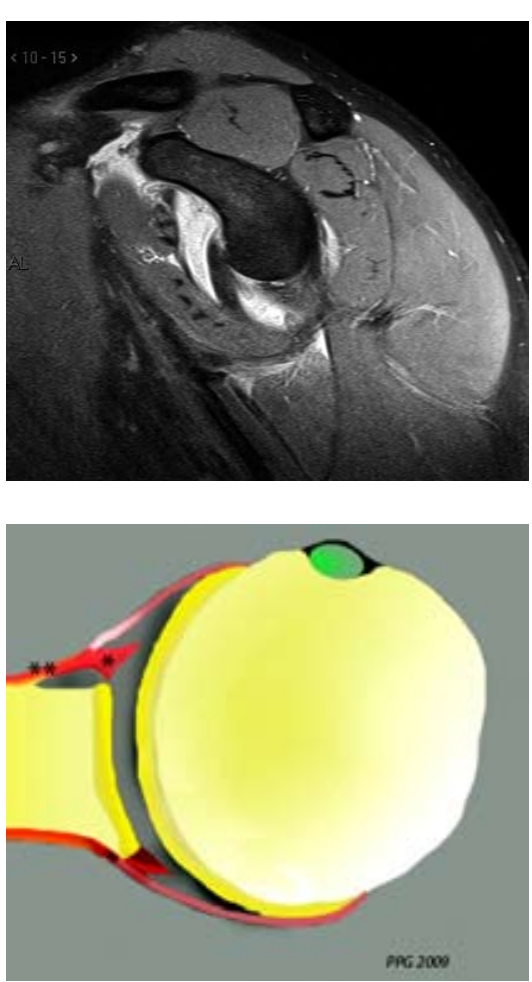

Figura 11a. Esquema lesión PERTHES: Desprendimiento del labrum anterior $\left(^{*}\right)$ desde el glenoides, con despegamiento, pero preservación de la continuidad del periostio $\left({ }^{* *}\right)$.

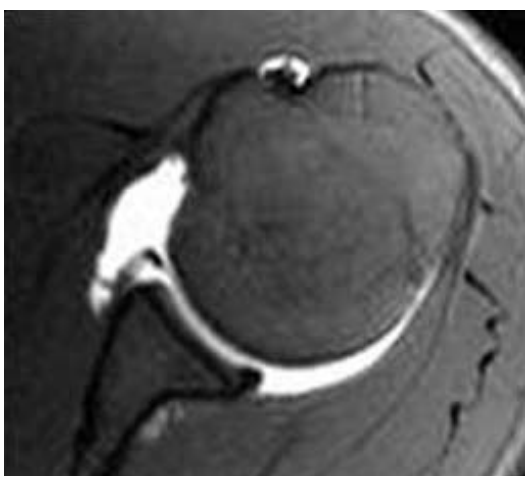

Figura 11 b. ArtroRM corte axial. Lesión de Perthes.

\section{Lesiones ligamentosas, condrales y otras}

Las roturas ligamentosas pueden asociarse a las lesiones labrales, especialmente aquellas que afectan al ligamento glenohumeral inferior.

\section{a. HAGL (humeral avulsion of the glenohumeral ligament)}

Consiste en un desgarro del ligamento glenohumeral inferior, en el sitio de su inserción en el húmero ${ }^{(10,11,19,20)}$. Puede asociarse o no a lesión tipo Bankart.

En artro-RM puede identificarse cuando existe un receso axilar en forma de "J" y extravasación del medio de contraste a dicho nivel (Figura 12).

Una variante de esta lesión resulta cuando se produce avulsión de un pequeño fragmento del húmero en relación a la inserción del ligamento glenohumeral inferior; se conoce con la sigla BHAGL (bone humeral avulsion of glenohumeral ligament) $)^{(10,11,19,20)}$.

b. GLAD (glenoid labrum articular disruption) También se le conoce con la sigla GARD (glenoid articular rim divot). Se define como un desgarro parcial del labrum glenoideo anterior, con un defecto condral en el cartílago articular glenoideo adyacente ${ }^{(12,19,20)}$.

Aunque esta lesión no deriva generalmente de luxación glenohumeral traumática, pueden verse lesiones similares en pacientes con inestabilidad ánteroinferior cuando se les evalúa con artro-RM (Figura 13).

c. Desgarro del ligamento glenohumeral inferior (IGL)

En esta lesión existe discontinuidad de las fibras del ligamento glenohumeral inferior en su porción media (intrasustancia). En la artro-RM se observa extravasación del medio de contraste a través del desgarro que se extiende inferiormente hacia el receso axilar (Figuras 14, 15). Los hallazgos se visualizan en el plano coronal.

Figura 12 a. ArtroRM corte coronal. Lesión HAGL.

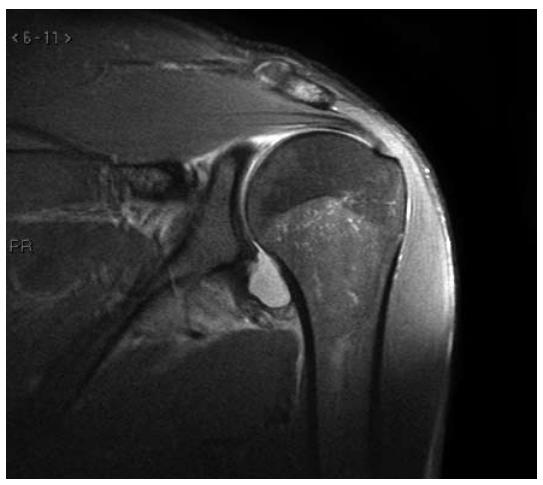

Figura 12 b. El mismo paciente de la figura anterior.
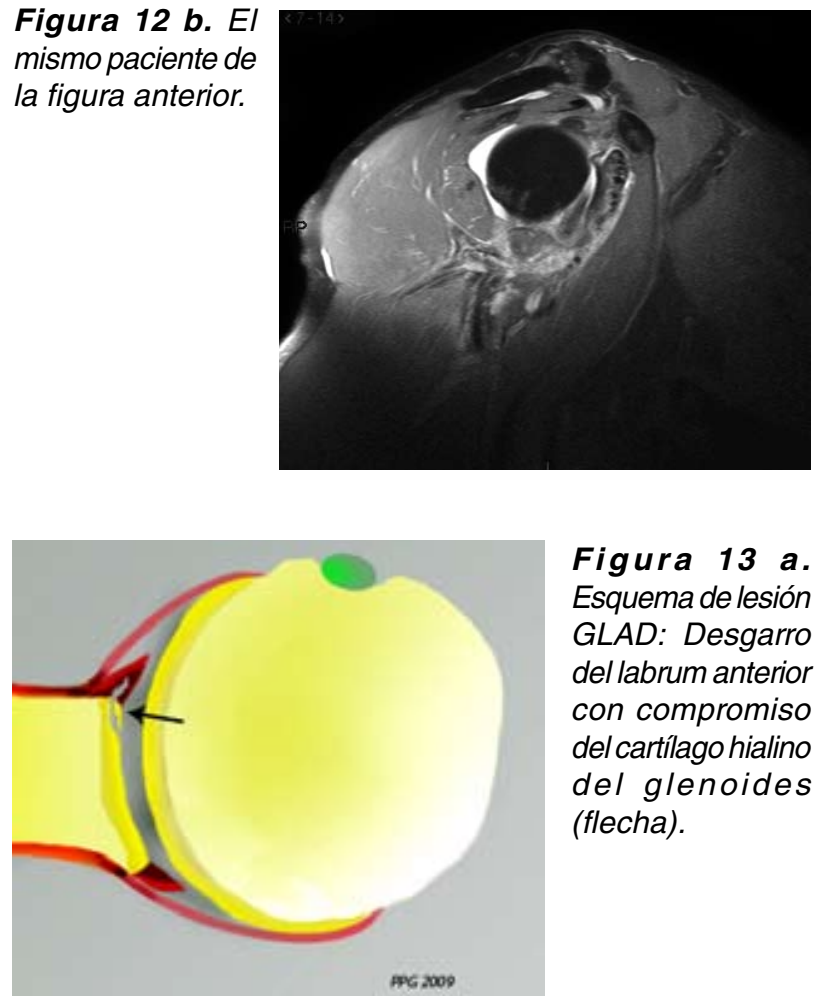

Figura 13 a. Esquema de lesión GLAD: Desgarro del labrum anterior con compromiso del cartílago hialino del glenoides (flecha). 


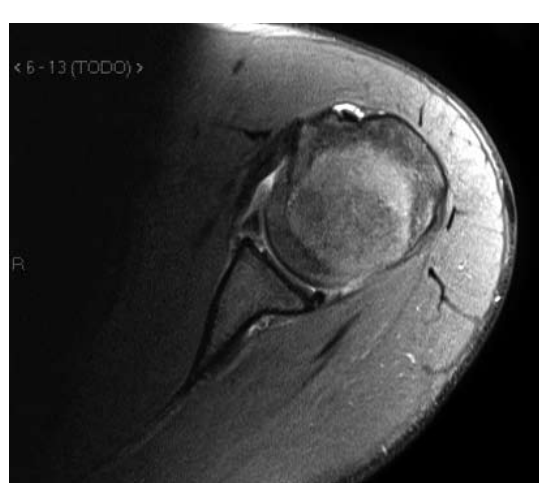

Figura 13 b. $R M$ corte axial DP con saturación de la grasa. Lesión GLAD.

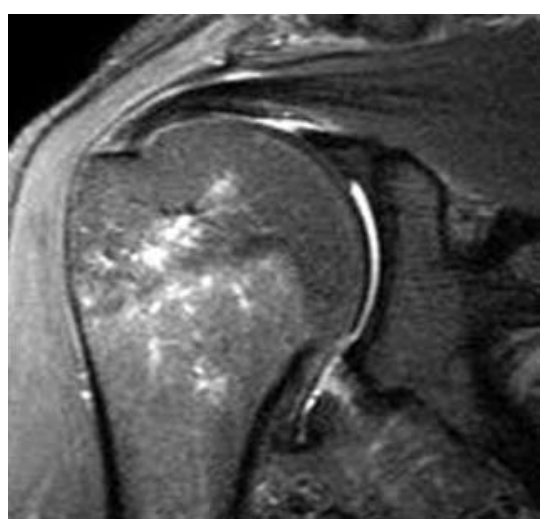

Figura 14. $R M$ corte coronal STIR. Desgarro del ligamento glenohumeral inferior.

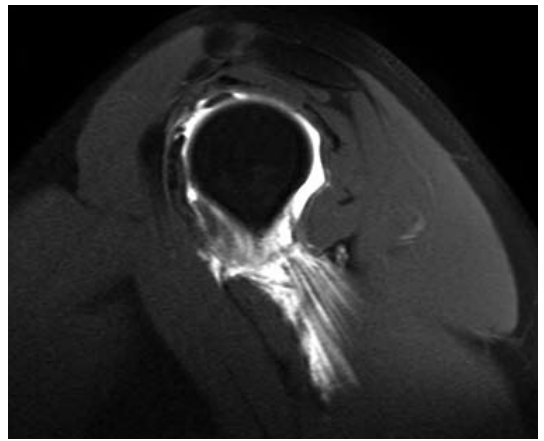

Figura 15. ArtroRM corte sagital. Desgarro del ligamento glenohumeral inferior.

4. Otras lesiones

a. Artrosis glenohumeral secundaria

Especialmente grave, se presenta tras episodios de luxación traumática posterior o determinados procedimientos quirúrgicos.

\section{b. Quistes paralabrales}

Se asocian con desgarros labrales, de hecho suelen originarse a partir de un desgarro del labrum. Su detección es difícil en las secuencias T1 convencionales de la artro-RM, dado que pueden no comunicar con la cavidad articular, razón por la que se debe incluir alguna secuencia sensible al líquido en el protocolo de artro-RM (Figura 16).

\section{c. Cuerpos intraarticulares}

Se observan con frecuencia en estos pacientes, tanto en los estudios por imágenes, específicamente los artrográficos (artroRM y
artroTC), como en artroscopía. Generalmente derivan de desgarros condrales o labrales. No deben ser confundidos con aire intraarticular, especialmente en la evaluación con artro-RM (Figura 17a y 17 b).

Figura 16 a. $R M$ corte sagital DP con saturación de la grasa. Quiste perilabral.

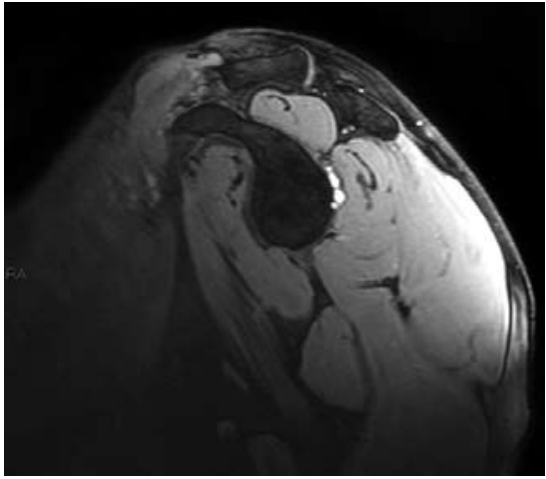

Figura 16 b. $R M$ corte axial. DP con saturación de la grasa. El mismo paciente de la figura anterior.

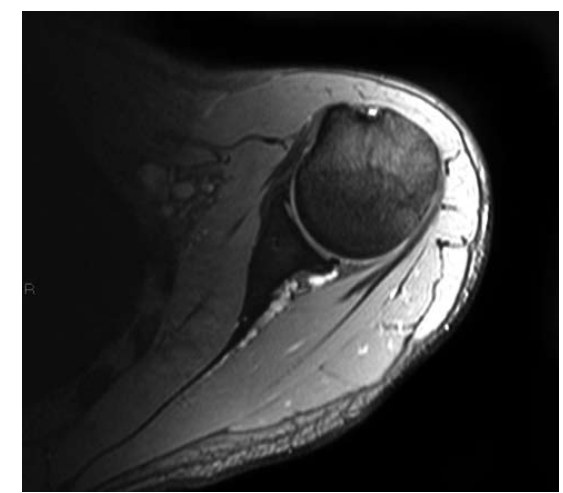

Figura 17 a. ArtroRM corte sagital. Cuerpo condral intraarticular.
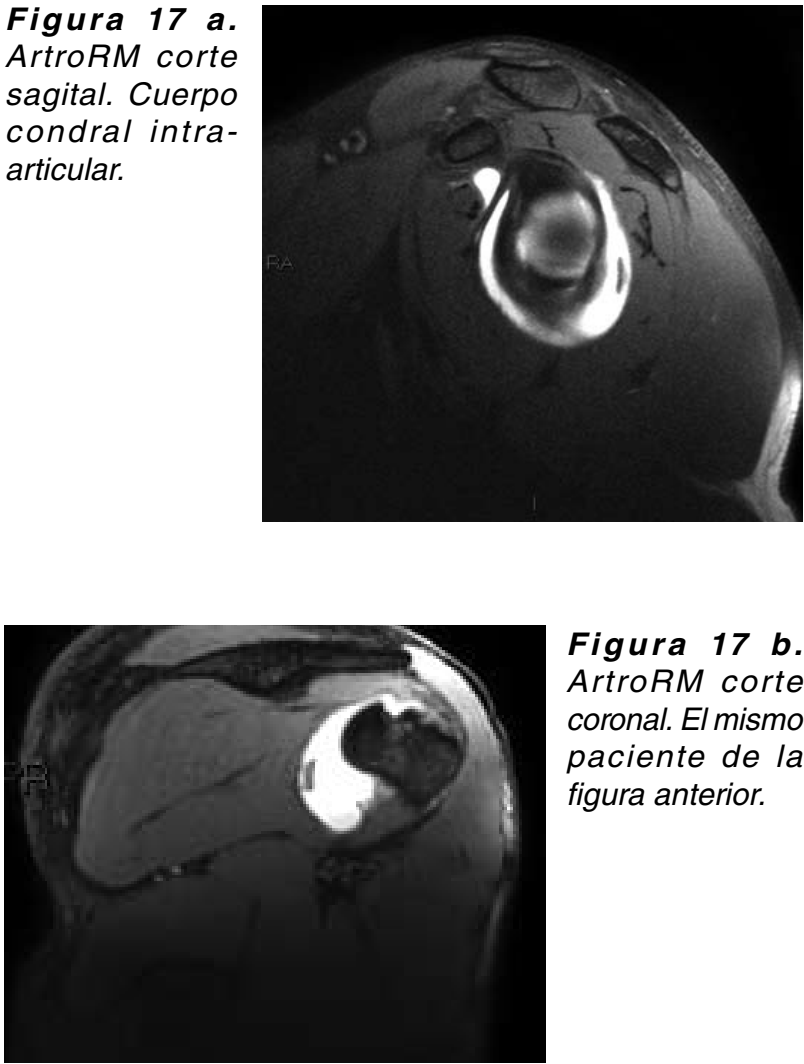

Figura 17 b. ArtroRM corte coronal. El mismo paciente de la figura anterior. 


\section{Luxación traumática posterior}

Puede ser secundaria a una contracción muscular violenta, habitualmente en el contexto de una crisis convulsiva o una descarga eléctrica. Ocurre también en atletas, como resultado de un fuerte traumatismo ánteroposterior.

En el estudio imaginológico, estos pacientes pueden mostrar diversas lesiones, incluyendo desgarro labral o cápsulo-labral posterior, lesión capsular, erosiones, esclerosis o formación de hueso heterotópico (lesión de Bennett) y fracturas de Mc Laughlin o Hill-Sachs reverso (Figura 18)(13,14,19,20).

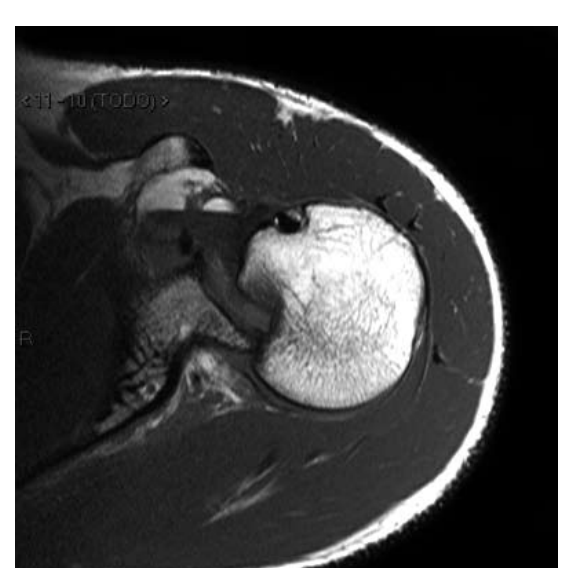

Figura 18 a. $R M$ corte axial T1. Fractura de Hill Sachs reversa.

Figura 18 b. $R M$ corte sagital T1. El mismo paciente de la figura anterior.

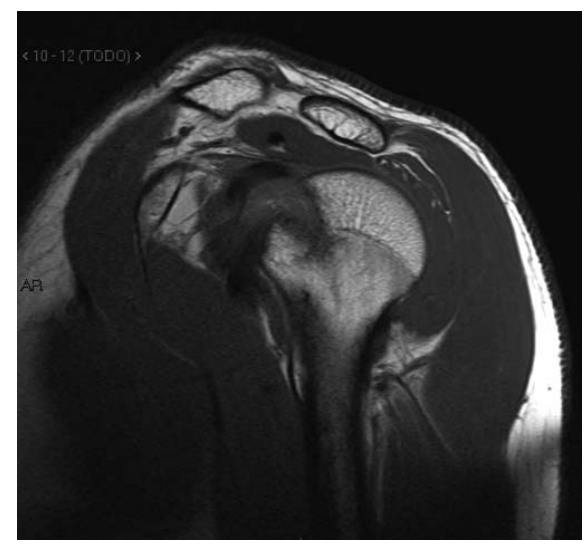

\section{a. Lesión de Bennet}

Osificación heterotópica extraarticular en el aspecto posterior de la articulación glenohumeral asociado a desgarro labral posterior y cambios inflamatorios-fibrosos en la cápsula articular (Figura 19) ${ }^{(14,19,20)}$

\section{b. Desgarro labral posterior}

También conocido como Bankart reverso, corresponde a una lesión del anillo glenoideo/cápsula/labrum posterior, secundario a una luxación posterior de hombro (Figura 20)(13-15,19,20).

Existe una variante del desgarro labral posterior clásico o Bankart reverso, que es la lesión POLPSA (posterior labrocapsular sleeve avulsion). A diferen- cia del Bankart reverso, la cápsula articular no está interrumpida. Corresponde a la misma alteración anatómica que la lesión ALPSA, pero en el complejo cápsulo-labral posterior ${ }^{(22)}$.

Figura 19. Esquema lesión de Bennet.
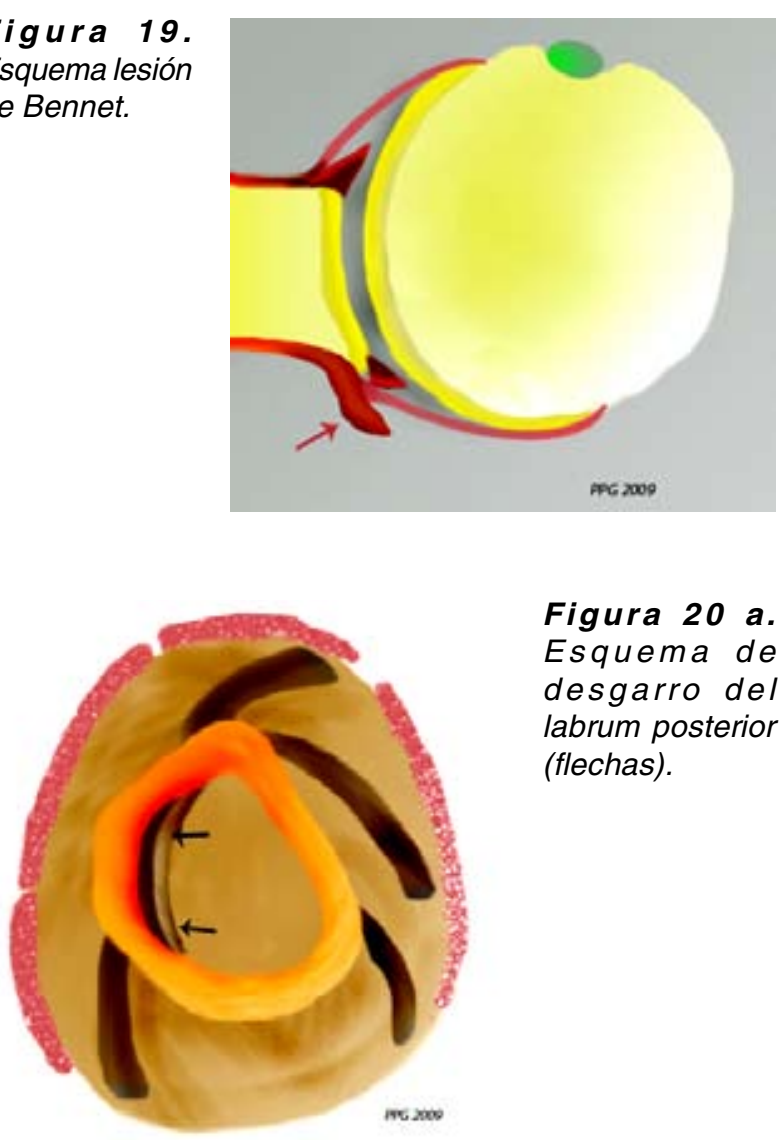

Figura 20 a. Esquema de desgarro del labrum posterior (flechas).

Figura 20 b. ArtroRM corte axial: Desgarro labral posterior.

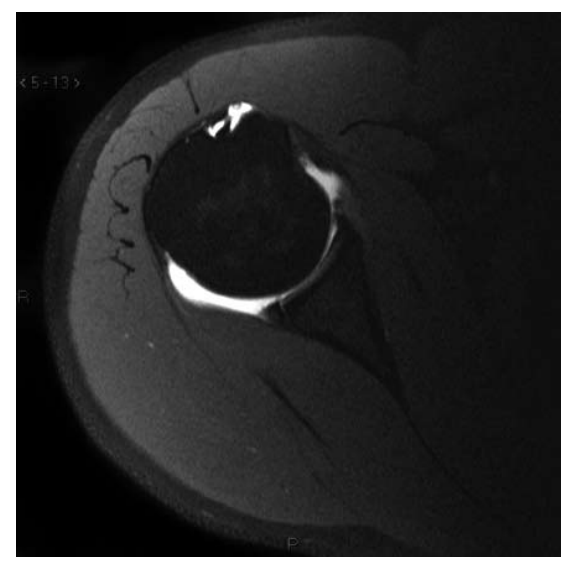

\section{Inestabilidad multidireccional}

Un porcentaje pequeño de los casos de inestabilidad glenohumeral son atraumáticos, multidireccionales y bilaterales ${ }^{(2-6)}$.

Su manejo terapéutico implica rehabilitación, aunque puede ser necesaria la cirugía, que muchas veces consiste en la reducción capsular. 
La inestabilidad multidireccional puede cursar sin hiperlaxitud y generalmente ocurre como consecuencia de al menos dos episodios previos de luxación traumática, con lesiones cápsulo-labrales anterior y posterior. Cuando cursa con hiperlaxitud, se relaciona con amplios recesos capsulares y con un labrum deficiente. Muchos de los casos de inestabilidad considerados multidireccionales corresponden en realidad a inestabilidad unidireccional asociada a hiperlaxitud; su interpretación errónea es perjudicial para un adecuado manejo quirúrgico.

Algunas variantes anatómicas de la escápula, tales como displasia glenoidea, deficiencia del margen pósteroinferior de la glenoides o retroversión glenoidea acentuada, pueden asociarse con mayor probabilidad a inestabilidad multidireccional(15).

\section{Microinestabilidad}

En el atleta, el microtraumatismo repetitivo puede generar elongación ligamentosa e hiperlaxitud adquirida. El término microinestabilidad, recientemente acuñado, se refiere al síndrome doloroso ánterosuperior acompañado de limitación funcional en el hombro atlético. En la microinestabilidad no existe antecedente de luxación glenohumeral documentada(2-6).

En estos pacientes pueden existir diversas lesiones menores, que también pueden estar presentes en aquellos con antecedentes de luxación glenohumeral ánteroinferior, incluyendo lesiones del labrum superior (tipo SLAP), especialmente del labrum pósterosuperior, lesiones del labrum superior asociadas con lesiones del manguito (tipo SLAC), lesiones del intervalo, roturas de la superficie articular del supraespinoso y lesiones del ligamento glenohumeral superior.

\section{Entidades relacionadas con la inestabilidad glenohumeral}

a. Lesiones del complejo labro-bicipital. SLAP (superior labral anterior to posterior lesions)

Afectan al complejo labro-bicipital en el sitio de inserción del tendón del bíceps; el labrum adyacente a esta inserción también puede estar comprometido, hacia adelante o hacia atrás. Resultan de fuerzas de compresión (28\%), caída con el brazo en extensión (22\%), tracción repentina $(25 \%)$ y sobreuso $(25 \%)^{(16-20)}$. Pueden ocurrir durante la práctica deportiva (movimientos del brazo sobre la cabeza) o por caída con el brazo en abducción. Clínicamente se presentan con dolor y sensación de "click"(16-18).

Inicialmente Snyder describió cuatro tipos, basado en los hallazgos artroscópicos. En series recientes, las lesiones SLAP han sido encontradas en un $36 \%$ de los pacientes con historia clínica de inestabilidad o dolor crónico de hombro, siendo el más frecuente el tipo II, encontrada en aproximadamente un $47 \%$ de los casos (Figura 21). En la actualidad se conocen muchos más tipos; se han descrito combinaciones de los anteriores y otros menos comunes que involucran labrum anterior o ligamento glenohumeral. Una alternativa a las diferentes clasificaciones para describir las lesiones SLAP es utilizar un método descriptivo de los desgarros del labrum, basándose en la circunferencia de un reloj e indicando la extensión de la lesión, utilizando como referencia las horas del reloj y tomando como hora 12 el sitio de inserción del tendón del bíceps (Figura 22).

Estas lesiones pueden asociarse con lesiones cápsulo-labrales anteriores y posteriores, causar inestabilidad o derivar de inestabilidad preexistente.

Figura 21 a. En verde se muestra el epicentro de la lesión SLAP.

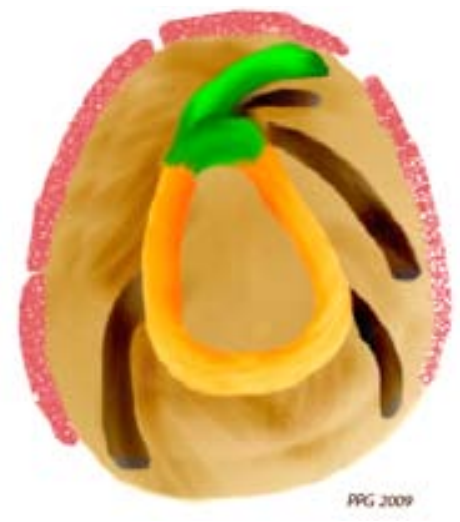

Figura 21 b. $R M$ corte coronal DP con saturación de la grasa. Lesión SLAP.

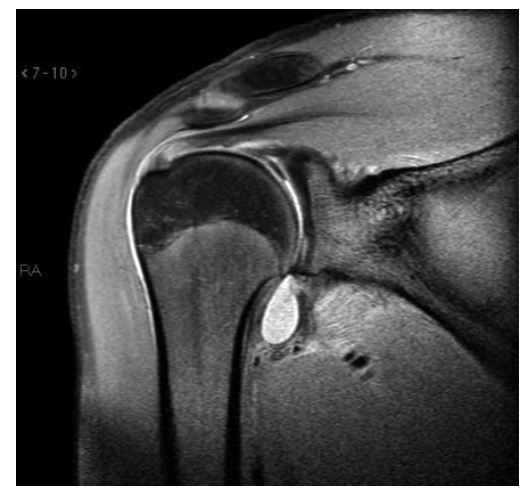

Figura 21 c. $R M$ corte sagital DP con saturación de la grasa. Hemartrosis. Lesión SLAP.

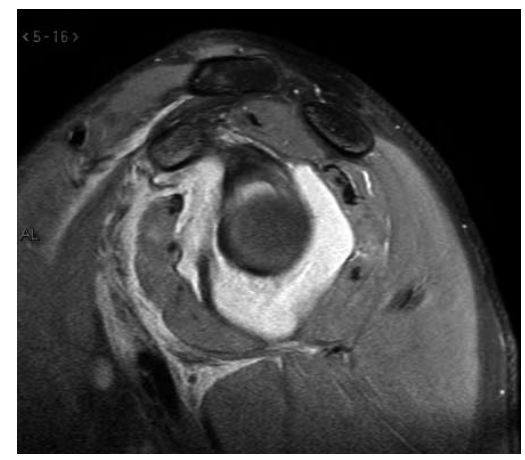

\section{b. Lesiones del intervalo rotador (hidden le sions)}

Las roturas del intervalo pueden derivar de luxación glenohumeral ánteroinferior o de 


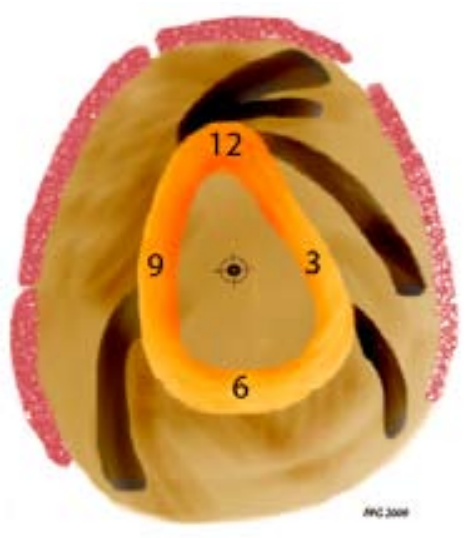

Figura 22. Representación esquemática de la glenoides de acuerdo al reloj. La hora 3 siempre es hacia anterior.

sobrecarga repetitiva en el hombro del atleta, encontrándose por lo tanto en el espectro de la microinestabilidad ${ }^{(19,20)}$.

Las roturas del intervalo pueden agravar la sintomatología de lesiones cápsulo-labrales preexistentes, añadiendo hiperlaxitud a una inestabilidad unidireccional y pueden cursar también como lesiones aisladas que generan dolor e inestabilidad inferior.

En artro-RM, las roturas del intervalo son más evidentes que en estudios de RM convencional. Se observa discontinuidad de los ligamentos coracohumeral y glenohumeral superior, lo cual genera extravasación del contraste.

\section{c. Tenosinovits bicipital}

El tendón de la porción larga del bíceps es un estabilizador secundario de la articulación glenohumeral y por lo tanto, todas aquellas causas que generan inestabilidad articular determinan estrés reiterado sobre el tendón, lo que puede desencadenar una tenosinovitis secundaria.

\section{d. Pinzamiento pósterosuperior}

Es un tipo de pinzamiento interno. Se trata de un cuadro típico del atleta lanzador o de deportistas que ocupan el brazo sobre la cabeza (nadadores y tenistas, por ejemplo). Se plantea que resultaría de una compresión repetitiva de la superficie articular de los tendones del manguito rotador (supraespinoso e infraespinoso) y la tuberosidad mayor del húmero contra el labrum glenoideo pósterosuperior. Se asocia a inestabilidad e hiperlaxitud cápsulo-ligamentosa anterior.

Clínicamente, se manifiesta como un síndrome doloroso del hombro que se presenta con roturas parciales de la superficie articular de las fibras posteriores del supraespinoso y/o anteriores del infraespinoso, generalmente asociadas a roturas del labrum pósterosuperior.

El uso de artro-RM, particularmente en la posición de abducción y rotación externa (ABER), resulta especialmente útil en estos pacientes. Los hallazgos incluyen: rotura parcial de la superficie articular de las fibras posteriores del supraespinoso y/o anteriores del infraespinoso, cambios degenerativos en el labrum pósterosuperior, desgarros del labrum pósterosuperior y alteraciones en el aspecto posterior de la tuberosidad mayor como quistes subcorticales, indentación focal y edema medular focal.

\section{Comentario final}

La inestabilidad glenohumeral es una causa frecuente de dolor y limitación funcional. La particular anatomía de la articulación glenohumeral condiciona o favorece la pérdida de la congruencia articular y determina inestabilidad del hombro.

Existe una clasificación clínico-radiológica que permite agrupar las causas que originan esta entidad y sus hallazgos radiológicos característicos.

Los métodos de estudio imaginológico actualmente disponibles son útiles para el diagnóstico de estas lesiones y su indicación dependerá del tipo de lesión que se sospeche, siendo sin lugar a dudas la Artro-RM la que ha emergido como la mejor técnica preoperatoria, pues presenta una mayor sensibilidad en el diagnóstico, especialmente de las lesiones intraarticulares, en comparación con la RM y la artro-TAC.

\section{Bibliografía}

1. Beltran J, Hyun-Min D. MR imaging of shoulder instability injuries in the athlete. Magn Reson Imaging Clin N Am 2003; 11: 221-238.

2. Justin Q. Ly, Douglas P. Beall, Timothy G. Sanders. MR Imaging of Glenohumeral Instability. AJR Am J Roentgenol 2003; 181: 203-213.

3. Gerber C, Nyffeler RW. Classification of glenohumeral joint instability. Clin Orthop 2002; 400: 65-76.

4. Shankman S, Bencardino J, Beltran J. Glenohumeral instability: evaluation using MR arthrography of the shoulder. Skeletal Radiol 1999; 28: 365-382.

5. Blum A, Coudane H, Mole D. Glenohumeral instabilities. Eur Radiol 2000; 10: 63-82.

6. Farber JM, Buckwalter KA. Sports-related injuries of the shoulder: instability. Radiol Clin North Am 2002; 40: 235-249.

7. Palmer W, Caslowitz P. Anterior shoulder instability: diagnostic criteria determined from prospective analysis of 121 MR arthrograms. Radiology 1995; 197: 819825.

8. Wischer TK, Bredella MA, Genant HK, Stoller DW, Bost FW, Tirman PFJ. Perthes lesion (a variant of the Bankart lesion): MR imaging and MR arthrographic findings with surgical correlation: AJR Am J Roentgenol 2002; 178: 233-237.

9. Burkhart SS, De Beer JF. Traumatic glenohumeral bone defects and their relationship to failure of arthroscopic Bankart repairs: significance of the inverted-pear glenoid and the humeral engaging Hill-Sachs lesion. Arthroscopy 2000; 16(7): 677-694.

10. Bui-Mansfield LT, Taylor DC, Uhorchak JM, Tenuta 
JJ. Humeral avulsions of the glenohumeral ligament: imaging features and review of the literature. AJR Am J Roentgenol 2002; 179: 649-655.

11. Tirman PFJ, Steinbach LS, Feller JF, Stauffer AE. Humeral avulsion of the anterior shoulder stabilizing structures after anterior shoulder dislocation: demonstration by MRI and MR arthrography. Skeletal Radiol 1996; 25: 743-748.

12. Neviaser TJ. The GLAD lesion: another cause of anterior shoulder pain. Arthroscopy 1993; 9: 22-23.

13. Yu JS, Ashman CJ, Jones G. The POLPSA lesion: MR imaging findings with arthroscopic correlation in patients with posterior instability. Skeletal Radiol 2002; 31: 396-399.

14. De Maeseneer M, Jaovissidha S, Jacobson JA, et al. The Bennett lesion of the shoulder. J Comput Assist Tomogr 1998; 2: 205-216.

15. Yu JS, Ashman CJ, Jones G. The POLPSA lesion: $\mathrm{MR}$ imaging findings with arthroscopic correlation in patients with posterior instability. Skeletal Radiol 2002; 31: 396-399.

16. Weishaupt D, Zanetti M, Nyffeler RW, Gerber C, Hodler J. Posterior glenoid rim deficiency in recurrent (atraumatic) posterior shoulder instability. Skeletal Radiol 2000; 29: 204-210.
17. Bencardino JT, Beltran J, Rosenberg ZS, Rokito A, Shankmann S, Mota J, et al. Superior labrum anteriorposterior lesions: diagnosis with MR arthrography of the shoulder. Radiology 2000; 214(1): 267-271.

18. Mohana-Borges AVR, Chung CB, Resnick D. Superior labral anteroposterior tear: classification and diagnosis on MRI and MR arthrography. AJR Am J Roentgenol 2003; 181: 1449-1462.

19. Waldt S, Burkat A, Lange $P$, et al. Diagnostic Performance of MR Arthrography in the Assessment of Superior labral Anteroposterior lesion of the shoulder. Am J Roentgenol AJR 2004; 182: 1271-78.

20. Stoller DW, Wolf EM. Shoulder. Stoller DW, Anderson LJ, Brody GA, Cannon WD, Ferkel RD, et al. Magnetic Resonance Imaging in Orthopaedics and Sports Medicine. 2nd Ed. Philadelphia. Lippincott-Raven; 1997; pp. 597-742.

21. Stoller DW, Tirman PFJ, Bredella MA. Shoulder. Stoller DW, Tirman PFJ, Bredella MA. Diagnostic Imaging Orthopaedics. 1st ed. Salt Lake Cyty, Utah. Amirsys; 2004; pp. 1-153.

22. Yu JS, Ashman CJ, Jones G. The POLPSA lesion: MR imaging with arthroscopic correlation in patients with posterior instability. Skeletal Radiol 2002; 31 : 396-399. 